Article

\title{
Generalized Abel-Grassmann's Neutrosophic Extended Triplet Loop
}

\author{
Xiaogang An ${ }^{1, *}$, Xiaohong Zhang ${ }^{1}$ and Yingcang $\mathrm{Ma}^{2}$ \\ 1 School of Arts and Sciences, Shaanxi University of Science \& Technology, Xi'an 710021, China; \\ zhangxiaohong@sust.edu.cn \\ 2 School of Science, Xi'an Polytechnic University, Xi'an 710048, China; mayingcang@xpu.edu.cn \\ * Correspondence: anxiaogang@sust.edu.cn
}

Received: 5 November 2019; Accepted: 4 December 2019; Published: 9 December 2019

\begin{abstract}
A group is an algebraic system that characterizes symmetry. As a generalization of the concept of a group, semigroups and various non-associative groupoids can be considered as algebraic abstractions of generalized symmetry. In this paper, the notion of generalized Abel-Grassmann's neutrosophic extended triplet loop (GAG-NET-Loop) is proposed and some properties are discussed. In particular, the following conclusions are strictly proved: (1) an algebraic system is an AG-NET-Loop if and only if it is a strong inverse AG-groupoid; (2) an algebraic system is a GAG-NET-Loop if and only if it is a quasi strong inverse AG-groupoid; (3) an algebraic system is a weak commutative GAG-NET-Loop if and only if it is a quasi Clifford AG-groupoid; and (4) a finite interlaced AG-(1,1)-Loop is a strong AG-(1,1)-Loop.
\end{abstract}

Keywords: Abel-Grassmann's neutrosophic extended triplet loop; generalized Abel-Grassmann's neutrosophic extended triplet loop; strong inverse AG-groupoid; quasi strong inverse AG-groupoid; quasi Clifford AG-groupoid

\section{Introduction}

The concept of an Abel-Grassmann's groupoid (AG-groupoid) was first given by Kazim and Naseeruddin [1] in 1972 and they have called it a left almost semigroup (LA-semigroup). In [2], the same structure is called a left invertive groupoid. In [3-9], some properties and different classes of an AG-groupoid are investigated.

Smarandache proposed the new concept of neutrosophic set, which is an extension of fuzzy set and intuitionistic fuzzy set [10]. Until now, neutrosophic sets have been applied to many fields such as decision making [11-13], forecasting [14], best product selection [15], the shortest path problem [16], minimum spanning tree [17], neutrosophic portfolios of financial assets [18], etc. Some new theoretical studies are also developed [19-24]. In [25], Xiaohong Zhang introduced the concept of Abel-Grassmann's neutrosophic extended triplet loop (AG-NET-loop), and some properties and structure about AG-NET-loop are discussed. Recently, a new algebraic system, generalized neutrosophic extended triplet set, is proposed in [26].

In this paper, we combine the notions of generalized neutrosophic extended triplet set and AG-groupoid, introduce the new concept of generalized Abel-Grassmann's neutrosophic extended triplet loop (GAG-NET-loop); that is, GAG-NET-loop is both AG-groupoid and generalized neutrosophic extended triplet set. We deeply analyze the internal connecting link between GAG-NETloop and other AG-groupoid and obtain some important results.

GAG-NET-loop is an extension of AG-NET-loop. Compared with AG-NET-loop, GAG-NET-loop relaxes the restriction on the elements in the AG-groupoid. According to our research, corresponding to the decomposition theorem of AG-NET-loop, some GAG-NET-loops can also be decomposed 
into smaller ones. This is also the embodiment of the research method of regular semigroups to quasi-regular semigroups in non-associative groupoid.

The paper is organized as follows. Section 2 gives the basic definitions. Some properties about finite interlaced AG-(1,1)-Loop and some structures about strong inverse AG-groupoid are discussed in Section 3. We proposed the GAG-NET-Loop and discussed its properties and structure in Section 4. Finally, the summary and future work are presented in Section 5.

\section{Basic Definitions}

In this section, the related research and results of the AG-NET-loop are presented. Some related notions are introduced first.

Let $S$ be non-empty set, $*$ is a binary operation on $S$. If $\forall a, b \in S$, implies $a * b \in S$, then $(S, *)$ is called a groupoid. A groupoid $(S, *)$ is called an Abel-Grassmann's groupoid (AG-groupoid) $[27,28]$ if it holds the left invertive law, that is, for all $a, b, c \in S,(a * b) * c=(c * b) * a$. In an AG-groupoid the medial law holds, for all $a, b, c, \in S,(a * b) *(c * d)=(a * c) *(b * d)$. In an AG-groupoid $(S, *)$, for all $a \in S, n \in Z^{+}$, the recursive definition of $a^{n}$ is as follows: $a^{1}=a, a^{2}=a * a, a^{3}=a^{2} * a=$ $(a * a) * a, a^{4}=a^{3} * a, \ldots, a^{n}=a^{n-1} * a$.

Definition 1 ([29]). Let $N$ be a non-empty set together with a binary operation $*$. Then, $N$ is called a neutrosophic extended triplet set if for any $a \in N$, there exists a neutral of " $a$ " (denoted by neut $(a)$ ), and an opposite of " $a$ "(denoted by anti $(a))$, such that neut $(a) \in N$, anti $(a) \in N$ and:

$$
\begin{gathered}
a * \operatorname{neut}(a)=\operatorname{neut}(a) * a=a, \\
a * \operatorname{anti}(a)=\operatorname{anti}(a) * a=\operatorname{neut}(a) .
\end{gathered}
$$

The triplet $(a, n e u t(a)$, anti $(a))$ is called a neutrosophic extended triplet.

Note that, for a neutrosophic triplet set $(N, *), a \in N$, neut $(a)$ and anti(a) may not be unique. In order not to cause ambiguity, we use the following notations to distinguish: neut (a) denotes any certain one of neutral of $a,\{$ neut $(a)\}$ denotes the set of all neutral of $a$, anti( $a)$ denotes any certain one of opposite of $a$, and $\{\operatorname{anti}(a)\}$ denotes the set of all opposite of $a$.

Definition 2 ([25]). Let $(N, *)$ be a neutrosophic extended triplet set. Then, $N$ is called a neutrosophic extended triplet loop (NET-Loop), if $(N, *)$ is well-defined, i.e., for any $a, b \in N$, one has $a * b \in N$.

Definition 3 ([25]). Let $(N, *)$ be a neutrosophic extended triplet loop (NET-Loop). Then, $N$ is called an AG-NET-Loop, if $(N, *)$ is an AG-groupoid.

An AG-NET-Loop $N$ is called a commutative AG-NET-Loop if for all $a, b \in N, a * b=b * a$.

Theorem 1 ([25]). Let $(N, *)$ be an AG-NET-loop. Then, for any $x, y \in\{\operatorname{anti}(a)\}$,

(1) $\operatorname{neut}(a) * x=x *$ neut $(a)=\operatorname{neut}(a) * y$, that is, $\mid$ neut $(a) *\{\operatorname{anti}(a)\} \mid=1$.

(2) $(x * \operatorname{neut}(a)) * a=($ neut $(a) * x) * a=\operatorname{neut}(a)$.

(3) $a *(x * \operatorname{neut}(a))=a *(\operatorname{neut}(a) * x)=\operatorname{neut}(a)$.

(4) $\forall a \in N$, neut $(a) * \operatorname{neut}(a)=\operatorname{neut}(a)$.

Definition 4 ([5]). An element a of an AG-groupoid $(S, *)$ is called a regular if there exists $x \in S$ such that $a=(a * x) * a$ and $S$ is called regular if all elements of $S$ are regular.

An $A G$-groupoid $(S, *)$ is called quasi regular if, for any $a \in S$, there exists a positive integer $n$ such that $a^{n}$ is regular. 
Definition 5 ([6]). An element a of an AG-groupoid $(S, *)$ is called a fully regular element of $S$ if there exist some $p, q, r, s, t, u, v, w, x, y, z \in S(p, q, \ldots, z$ may be repeated) such that

$$
\begin{aligned}
a & =\left(p * a^{2}\right) * q=(r * a) *(a * s)=(a * t) *(a * u) \\
& =(a * a) * v=w *(a * a)=(x * a) *(y * a) \\
& =\left(a^{2} * z\right) * a^{2} .
\end{aligned}
$$

An AG-groupoid $(S, *)$ is called fully regular if all elements of $S$ are fully regular.

An AG-groupoid $(S, *)$ is called quasi fully regular if for any $a \in S$, there exists a positive integer $n$ such that $a^{n}$ is fully regular.

\section{Strong Inverse AG-Groupoid and Finite Interlaced AG-Groupoid}

Definition 6 ([30]). An AG-groupoid $(S, *)$ is called an inverse AG-groupoid if for each element $a \in S$, there exists an element $x$ in $S$ such that $a=(a * x) * a$ and $x=(x * a) * x$.

Definition 7. An AG-groupoid $(S, *)$ is called a strong inverse AG-groupoid if for any a $\in S$, there exists a unary operation $a \rightarrow a^{-1}$ on $S$ such that

$$
\left(a^{-1}\right)^{-1}=a, \quad\left(a * a^{-1}\right) * a=a *\left(a * a^{-1}\right)=a, a * a^{-1}=a^{-1} * a .
$$

The following example shows that an inverse AG-groupoid may not be a strong inverse AG-groupoid.

Example 1. Let $S=\{1,2,3,4\}$, an operation $*$ on $S$ is defined as in Table 1 . Being $1=(1 * 3) * 1,3=$ $(3 * 1) * 3,2=(2 * 4) * 2,4=(4 * 2) * 4$, from Definition $6, S$ is an inverse AG-groupoid. Being $(1 * 1) * 1=$ $3 \neq 1,(1 * 2) * 1=4 \neq 1,(1 * 3) * 1=1 \neq 3=1 *(1 * 3),(1 * 4) * 1=2 \neq 1$, from Definition $7, S$ is not a strong inverse AG-groupoid.

Table 1. The operation table of Example 1.

\begin{tabular}{lllll}
\hline $\boldsymbol{*}$ & $\mathbf{1}$ & $\mathbf{2}$ & $\mathbf{3}$ & $\mathbf{4}$ \\
\hline 1 & 2 & 4 & 3 & 1 \\
2 & 3 & 1 & 2 & 4 \\
3 & 1 & 3 & 4 & 2 \\
4 & 4 & 2 & 1 & 3 \\
\hline
\end{tabular}

Proposition 1. Let $(N, *)$ be an AG-NET-loop. Then, for any $a \in N, x \in\{\operatorname{anti}(a)\}$,

$$
\operatorname{neut}(\operatorname{neut}(a) * x) * \operatorname{anti}(\operatorname{neut}(a) * x)=a \text {. }
$$

Proof. For any $x \in\{\operatorname{anti}(a)\}$, we have

$$
\begin{aligned}
(\text { neut }(a) * x) * \text { neut }(a) & =(\text { neut }(a) * x) *(a * x) \\
& =(\text { neut }(a) * a) *(x * x) \quad \text { (applying the medial law) } \\
& =(a * \operatorname{neut}(a)) *(x * x) \\
& =(a * x) *(\text { neut }(a) * x) \quad \text { (applying the medial law) } \\
& =\operatorname{neut}(a) *(\operatorname{neut}(a) * x),
\end{aligned}
$$




$$
\begin{aligned}
\text { neut }(a) *(\text { neut }(a) * x) & =(x * a) *(\text { neut }(a) * x) \\
& =(x * \text { neut }(a)) *(a * x) \quad \text { (applying the medial law) } \\
& =(x * \text { neut }(a)) * \text { neut }(a) \\
& =(\text { neut }(a) * \text { neut }(a)) * x \\
& =\text { neut }(a) * x, \quad(\text { by Proposition } 1(4))
\end{aligned}
$$

we have $(\operatorname{neut}(a) * x) * \operatorname{neut}(a)=\operatorname{neut}(a) *(\operatorname{neut}(a) * x)=\operatorname{neut}(a) * x$.

From Theorem 1 (2) and (3), we have

$$
\text { neut }(\text { neut }(a) * x)=\operatorname{neut}(a), a \in \operatorname{anti}\{\text { neut }(a) * x\} .
$$

From Theorem 1 (1) neut $(a) * x$ is unique, we have

$$
\operatorname{neut}(\text { neut }(a) * x) * \operatorname{anti}(\operatorname{neut}(a) * x)=\operatorname{neut}(a) * a=a
$$

Example 2. Let $N=\{a, b, c\}$, an operation $*$ on $N$ is defined as in Table 2. Since neut $(a)=a$, anti $(a)=$ $a$, neut $(b)=a, \operatorname{anti}(b)=c$, neut $(c)=a, \operatorname{anti}(c)=b$, so $(N, *)$ is an AG-NET-Loop. Being

$$
\begin{gathered}
\text { neut }(\text { neut }(a) * a) * \operatorname{anti}(\text { neut }(a) * a)=a * a=a, \\
\operatorname{neut}(\operatorname{neut}(b) * c) * \operatorname{anti}(\operatorname{neut}(b) * c)=\operatorname{neut}(c) * \operatorname{anti}(c)=b, \\
\operatorname{neut}(\operatorname{neut}(c) * b) * \operatorname{anti}(\operatorname{neut}(c) * b)=\operatorname{neut}(b) * \operatorname{anti}(b)=c,
\end{gathered}
$$

that is for any $a \in N, x \in\{\operatorname{anti}(a)\}$, neut $($ neut $(a) * x) * \operatorname{anti}(\operatorname{neut}(a) * x)=a$.

Table 2. An AG-NET-Loop of Example 2.

\begin{tabular}{llll}
\hline$*$ & $\boldsymbol{a}$ & $\boldsymbol{b}$ & $\boldsymbol{c}$ \\
\hline$a$ & $a$ & $b$ & $c$ \\
$b$ & $b$ & $c$ & $a$ \\
$c$ & $c$ & $a$ & $b$ \\
\hline
\end{tabular}

Theorem 2. Let $(N, *)$ be a groupoid. Then, $N$ is an AG-NET-Loop if and only if it is a strong inverse AG-groupoid.

Proof. Necessity: Suppose $N$ is an AG-NET-Loop, from Definition 3, for each $a \in N$, such that $a$ has the neutral element and opposite element, denoted by neut $(a)$ and anti(a), respectively. Set

$$
a^{-1}=\operatorname{neut}(a) * \operatorname{anti}(a),
$$

by Theorem $1(1)$, neut $(a) * \operatorname{anti}(a)$ is unique, so $a^{-1}$ is unique. By Proposition 1 , we have

$$
\left(a^{-1}\right)^{-1}=\operatorname{neut}(\text { neut }(a) * \operatorname{anti}(a)) * \operatorname{anti}(\text { neut }(a) * \operatorname{anti}(a))=a \text {. }
$$

Being

$$
a^{-1} * a=(\operatorname{neut}(a) * \operatorname{anti}(a)) * a=(a * \operatorname{anti}(a)) * \operatorname{neut}(a)=\operatorname{neut}(a) * \operatorname{neut}(a)=\operatorname{neut}(a),
$$




$$
\begin{aligned}
& a * a^{-1}=a *(\operatorname{neut}(a) * \operatorname{anti}(a)) \\
&=(\operatorname{neut}(a) * a) *(\operatorname{neut}(a) * \operatorname{anti}(a)) \\
&=(\operatorname{neut}(a) * \operatorname{neut}(a)) *(a * \operatorname{anti}(a)) \\
&=(\operatorname{neut}(a) * \operatorname{neut}(a)) * \operatorname{neut}(a) \\
&=\operatorname{neut}(a), \\
&\left(a * a^{-1}\right) * a=\operatorname{neut}(a) * a=a, \\
& a *\left(a * a^{-1}\right)=a * \operatorname{neut}(a)=a,
\end{aligned}
$$

we have

$$
\begin{gathered}
a^{-1} * a=a * a^{-1}, \\
\left(a * a^{-1}\right) * a=a *\left(a * a^{-1}\right)=a .
\end{gathered}
$$

From Definition 7, $\mathrm{N}$ is a strong inverse AG-groupoid.

Sufficiency: If $N$ is a strong inverse AG-groupoid and $a^{-1} \in N$, such that $a * a^{-1}=a^{-1} * a$ and $\left(a * a^{-1}\right) * a=a *\left(a * a^{-1}\right)=a$. Set

$$
\operatorname{neut}(a)=a * a^{-1},
$$

then $\operatorname{neut}(a) * a=\left(a * a^{-1}\right) * a=a *\left(a * a^{-1}\right)=a * \operatorname{neut}(a)=a, a *(a)^{-1}=(a)^{-1} * a=\operatorname{neut}(a)$. From Definition 3, we have that $N$ is an AG-NET-Loop and $a^{-1} \in\{\operatorname{anti}(a)\}$.

Example 3. Apply $(S, *)$ in Example 2, we know that it is an AG-NET-Loop. We show that it is a strong inverse AG-groupoid in the following.

For $b$, there exists a inverse element $b^{-1}=c$, such that $\left(b^{-1}\right)^{-1}=b,\left(b * b^{-1}\right) * b=b *\left(b * b^{-1}\right)=$ $b, b * b^{-1}=b^{-1} * b=a$, so $b$ is strong inverse. $a$ and $c$ are strong inverse for the same reason, so $(S, *)$ is a strong inverse AG-groupoid by Definition 7.

An AG-groupoid $(\mathrm{S}, *)$ is called interlaced if it satisfies $(a * a) * b=a *(a * b), a *(b * b)=(a * b) * b$ for all $a, b$ in $S$. An AG-groupoid $\left(\mathrm{S},{ }^{*}\right)$ is called locally associative if it satisfies $(a * a) * a=a *(a * a)$ for all $a$ in $S$.

Theorem 3. Let $(D, *)$ be a locally associative AG-groupoid with respect to *. If $D$ is finite, there is an idempotent element in $D$. That is, $\exists a \in D, a * a=a$.

Proof. Assume that $D$ is a finite locally associative AG-groupoid with respect to * Then, for any $a \in D$, $a, a * a=a^{2}, a * a * a=a^{3}, \ldots, a^{n}, \ldots \in D$. Since $D$ is finite, there exists natural number $m, k$ such that $a^{m}=a^{m+k}$.

Case 1: If $k=m$, then $a^{m}=a^{2 m}$, that is, $a^{m}=a^{m} * a^{m}, a^{m}$ is an idempotent element in $D$.

Case 2: If $k>m$, then from $a^{m}=a^{m+k}$ we can get

$$
a^{k}=a^{m} * a^{k-m}=a^{m+k} * a^{k-m}=a^{2 k}=a^{k} * a^{k} .
$$

This means that $a^{k}$ is an idempotent element in $D$.

Case 3: If $k<m$, then from $a^{m}=a^{m+k}$ we can get

$$
\begin{gathered}
a^{m}=a^{m+k}=a^{m} * a^{k}=a^{m+k} * a^{k}=a^{m+2 k} \\
a^{m}=a^{m+2 k}=a^{m} * a^{2 k}=a^{m+k} * a^{2 k}=a^{m+3 k} \\
\cdots \\
a^{m}=a^{m+m k} .
\end{gathered}
$$


Since $m$ and $k$ are natural numbers, then $m k \geq m$. Therefore, from $a^{m}=a^{m+m k}$, applying Case 1 or Case 2, we know that there exists an idempotent element in $D$.

Definition 8 ([31]). Let $(N, *)$ be an AG-groupoid. Then, $N$ is called an $A G-(l, l)$-Loop, if for any $a \in N$, there exist two elements $b$ and $c$ in $N$ that satisfy the condition: $b * a=a$, and $c * a=b$. In an $A G-(l, l)$-Loop, a neutral of " $a$ " denoted by neut $(l, l)(a)$.

Definition 9 ([31]). Let $(N, *)$ be an AG-(l, l)-Loop. Then, $N$ is a strong $A G-(l, l)$-Loop if neut $(l, l)(a) *$ $\operatorname{neut}_{(l, l)}(a)=\operatorname{neut}_{(l, l)}(a), \forall a \in N$.

Definition 10. Let $(D, *)$ be an AG- $(l, l)$-Loop. Then, $D$ is called an interlaced AG-(l,l)-Loop, if it satisfies $(a *$ $a) * b=a *(a * b), a *(b * b)=(a * b) * b$, for all $a, b$ in $D$.

Theorem 4. Let $(D, *)$ be an interlaced $A G-(l, l)$-Loop with respect to ${ }^{*}$. If $D$ is finite, there is an idempotent left neutral element in $D$. That is, $\forall a \in D, \exists s, p \in D, s * a=a, p * a=s, s * s=s$.

Proof. Assume that $D$ is a finite interlaced AG-(1,1)-Loop with respect to * Then, for any $a \in D$, $\exists s, p \in D, s * a=a, p * a=s$, we have $s * a=(p * a) * a=(a * a) * p=a *(a * p)=a$,

$$
\begin{aligned}
& a * s=(a *(a * p)) * s \\
&=(s *(a * p)) * a \quad \text { (by the left invertive law }) \\
&=((p * a) *(a * p)) * a \\
&=(((a * p) * a) * p) * a \quad \text { (by the left invertive law }) \\
&=(a * p) *((a * p) * a) \quad(\text { by the left invertive law }) \\
&=((a * p) *(a * p)) * a \quad \text { (by the interlaced law }) \\
&=(a *(a * p)) *(a * p) \quad(\text { by the left invertive law }) \\
&=a *(a * p)=a, \\
& s^{2} * a=(s * s) * a=(a * s) * s=a, \\
& s^{3} * a=\left(s^{2} * s\right) * a=(a * s) * s^{2}=a * s^{2}=a *(s * s)=(a * s) * s=a * s=a .
\end{aligned}
$$

When $m>3, m \equiv 0(\bmod 2)$, we have

$$
\begin{aligned}
s^{m} * a & =\left(s^{m-2} * s^{2}\right) * a \\
& =\left(a * s^{2}\right) * s^{m-2} \\
& =a * s^{m-2} \\
& =a *\left(s^{(m-2) / 2} * s^{(m-2) / 2}\right) \\
& \left.=\left(a * s^{(m-2) / 2}\right) * s^{(m-2) / 2} \quad \text { (by the interlaced law }\right) \\
& \left.=\left(s^{(m-2) / 2} * s^{(m-2) / 2}\right) * a \quad \text { (by the left invertive law }\right) \\
& =s^{m-2} * a \\
& =\ldots \ldots \\
& =s^{2} * a=a .
\end{aligned}
$$


When $m>3, m \equiv 1(\bmod 2)$, we have

$$
\begin{aligned}
s^{m} * a & =\left(s^{m-1} * s\right) * a \\
& =(a * s) * s^{m-1} \\
& =a * s^{m-1} \\
& =a *\left(s^{(m-1) / 2} * s^{(m-1) / 2}\right) \\
& \left.=\left(a * s^{(m-1) / 2}\right) * s^{(m-1) / 2} \quad \text { (by the interlaced law }\right) \\
& =\left(s^{(m-1) / 2} * s^{(m-1) / 2}\right) * a \\
& =s^{m-1} * a \\
& =\ldots . . \\
& =s^{2} * a=a .
\end{aligned}
$$

Thus, $s, s^{2}, s^{3} \ldots \ldots s^{m} \ldots \ldots$ are all left neutral element.

Applying Theorem 3, we know that there exists an idempotent left neutral element in $D$.

Theorem 5. Assume that $(N, *)$ is a finite interlaced $A G-(l, l)$-Loop. Then, for all a in $N$, if neut $(l, l)(a)$ is an idempotent element, then it is unique.

Proof. Assume that $N$ is a finite interlaced AG-(1,1)-Loop with respect to *. Suppose that there exist $x, y \in\left\{\right.$ neut $\left._{(l, l)}(a)\right\}, a \in N$. By Definition $8, x * a=a, y * a=a$, and there exist $p, q \in N$ which satisfy $p * a=x, q * a=y$. If $x * x=x, y * y=y$, we have

$$
\begin{gathered}
x=x * x=(p * a) * x=(x * a) * p=a * p, \\
y=y * y=(q * a) * y=(y * a) * q=a * q, \\
x * y=(p * a) * y=(y * a) * p=a * p=x, \\
y * x=(q * a) * x=(x * a) * q=a * q=y, \\
x=x * y=(x * x) * y=(y * x) * x=y * x=y .
\end{gathered}
$$

We know that $x=y$, neut $_{(l, l)}(a)$ is unique.

Theorem 6. Let $(N, *)$ be a finite interlaced AG- $(l, l)$-Loop. Then, $N$ is a strong $A G-(l, l)$-Loop.

Proof. For any $a$ in $N$, applying Theorem 4, we have $\exists s, p \in N, s * a=a, p * a=s, s * s=s$. From this and Definition 9, we know that $N$ is a strong AG-(1,1)-Loop.

Example 4. Let $S=\{1,2,3\}$, an operation $*$ on $S$ is defined as in Table 3 . Being $(1 * 1) * 2=1 *(1 * 2)=$ $2,1 *(2 * 2)=(1 * 2) * 2=3,(1 * 1) * 3=1 *(1 * 3)=3,1 *(3 * 3)=(1 * 3) * 3=2,(2 * 2) * 3=$ $2 *(2 * 3)=2,2 *(3 * 3)=(2 * 3) * 3=3$, and $1 * 1=1,1 * 2=2,3 * 2=1,1 * 3=3,2 * 3=1$, we have $S$ is a finite interlaced $A G-(l, l)$-Loop by Definition 10. Being neut $(l, l)(1)=$ neut $_{(l, l)}(2)=$ neut $_{(l, l)}(3)=1$, $1^{*} 1=1$, we have $S$ is a strong $A G-(l, l)$-Loop by Definition 9 .

Table 3. A finite interlaced AG-(1,1)-Loop of Example 4.

\begin{tabular}{llll}
\hline$*$ & $\mathbf{1}$ & $\mathbf{2}$ & $\mathbf{3}$ \\
\hline 1 & 1 & 2 & 3 \\
2 & 2 & 3 & 1 \\
3 & 3 & 1 & 2 \\
\hline
\end{tabular}


The following example shows that a strong AG-(1,1)-Loop may not be an interlaced AG-(1,1)-Loop.

Example 5. Let $S=\{1,2,3\}$, an operation $*$ on $S$ is defined as in Table 4. Being $1 * 1=1,1 * 2=2,2 * 2=$ $1,1 * 3=3,3 * 3=1$, we have $S$ is a strong $A G-(l, l)$-Loop by Definition 9. However, it is not an interlaced $A G-(l, l)$-Loop because $2 *(3 * 3)=3 \neq 2=(2 * 3) * 3$.

Table 4. A strong AG-(1,1)-Loop of Example 5.

\begin{tabular}{llll}
\hline $\boldsymbol{*}$ & $\mathbf{1}$ & $\mathbf{2}$ & $\mathbf{3}$ \\
\hline 1 & 1 & 2 & 3 \\
2 & 3 & 1 & 2 \\
3 & 2 & 3 & 1 \\
\hline
\end{tabular}

\section{GAG-NET-Loop}

Definition 11 ([26]). Let $N$ be a non-empty set together with a binary operation $*$. Then, $N$ is called a generalized neutrosophic extended triplet set if for any $a \in N$, at least exists a positive integer $n$, $a^{n}$ exists neutral element (denoted by neut $\left(a^{n}\right)$ ) and opposite element (denoted by anti $\left(a^{n}\right)$ ), such that $\operatorname{neut}\left(a^{n}\right) \in N$, anti $\left(a^{n}\right) \in N$ and

$$
a^{n} * \operatorname{neut}\left(a^{n}\right)=\operatorname{neut}\left(a^{n}\right) * a^{n}=a^{n}, a^{n} * \operatorname{anti}\left(a^{n}\right)=\operatorname{anti}\left(a^{n}\right) * a^{n}=\operatorname{neut}\left(a^{n}\right) .
$$

The triplet $\left(a, n e u t\left(a^{n}\right)\right.$, anti $\left.\left(a^{n}\right)\right)$ is called a generalized neutrosophic extended triplet with degree $n$.

Definition 12. Let $(N, *)$ be a generalized neutrosophic extended triplet set. Then, $N$ is called a generalized Abel-Grassmann's neutrosophic extended triplet loop (GAG-NET-Loop), if the following conditions are satisfied: for all $a, b, c \in N,(a * b) * c=(c * b) * a$.

A GAG-NET-Loop $N$ is called a commutative GAG-NET-Loop if for all $a, b \in N, a * b=b * a$.

Example 6. Let $S=\{a, b, c\}$, an operation $*$ on $S$ is defined as in Table 5. We can see that $(a, a, a),(a, a, b)$, and $(a, a, c)$ are neutrosophic extended triplets, but $b$ and $c$ do not have the neutral element and opposite element. Thus, $S$ is not an AG-NET-Loop. Moreover, $b^{2}=c^{2}=a$ has the neutral element and opposite element, thus $(S, *)$ is a GAG-NET-Loop. $(b, a, a)$ and $(c, a, a)$ are generalized neutrosophic extended triplets with degree 2. We can infer that $(S, *)$ is a GAG-NET-Loop but not an AG-NET-Loop. Moreover it is not a commutative GAG-NET-Loop being $b * c \neq c * b$.

Table 5. A GAG-NET-Loop of Example 6.

\begin{tabular}{llll}
\hline$*$ & $\boldsymbol{a}$ & $\boldsymbol{b}$ & $\boldsymbol{c}$ \\
\hline$a$ & $a$ & $a$ & $a$ \\
$b$ & $a$ & $a$ & $c$ \\
$c$ & $a$ & $b$ & $a$ \\
\hline
\end{tabular}

The algebraic system $\left(Z_{n}, \otimes\right), \otimes$ is the classical mod multiplication, where $Z_{n}=\{[0],[1], \cdots,[n-$ $1]\}$ and $n \in Z^{+}, n \geq 2$.

Example 7. Consider $\left(Z_{4}, \otimes\right)$, an operation $\otimes$ on $Z_{4}$ is defined as in Table 6 . We have:

(1) $[0],[1]$ and [3] have the neutral element and opposite element.

(2) [2] does not have the neutral element and opposite element, but we can see that $[2]^{2}=[0]$ has the neutral element and opposite element. 
Table 6. The operation table of $Z_{4}$.

\begin{tabular}{lllll}
\hline$\otimes$ & {$[0]$} & {$[1]$} & {$[2]$} & {$[3]$} \\
\hline$[0]$ & {$[0]$} & {$[0]$} & {$[0]$} & {$[0]$} \\
{$[1]$} & {$[0]$} & {$[1]$} & {$[2]$} & {$[3]$} \\
{$[2]$} & {$[0]$} & {$[2]$} & {$[0]$} & {$[2]$} \\
{$[3]$} & {$[0]$} & {$[3]$} & {$[2]$} & {$[1]$} \\
\hline
\end{tabular}

Thus, $Z_{4}$ is a generalized neutrosophic extended triplet set, but it is not a neutrosophic extended triplet set. Moreover, $\left(Z_{4}, \otimes\right)$ is a commutative GAG-NET-Loop.

Proposition 2. Let $(N, *)$ be a GAG-NET-Loop, $a \in N$ and $\left(a\right.$, neut $\left(a^{n}\right)$, anti $\left.\left(a^{n}\right)\right)$ is a generalized neutrosophic extended triplet with degree $n$. We have:

(1) neut $\left(a^{n}\right)$ is unique.

(2) neut $\left(a^{n}\right) *$ neut $\left(a^{n}\right)=\operatorname{neut}\left(a^{n}\right)$.

Proof. Assume $c, d \in\left\{\right.$ neut $\left.\left(a^{n}\right)\right\}$, so $a^{n} * c=c * a^{n}=a^{n}, a^{n} * d=d * a^{n}=a^{n}$, and there exists $x, y \in N$ such that

$$
a^{n} * x=x * a^{n}=c, a^{n} * y=y * a^{n}=d .
$$

We can obtain

$$
\begin{aligned}
& c * d=\left(x * a^{n}\right) * d=\left(d * a^{n}\right) * x=a^{n} * x=c, \\
& c * d=\left(a^{n} * x\right) *\left(y * a^{n}\right) \\
&=\left(a^{n} * y\right) *\left(x * a^{n}\right) \\
&=\left(a^{n} * y\right) * c \\
&=\left(y * a^{n}\right) * c \\
&=\left(c * a^{n}\right) * y \\
&=a^{n} * y=d .
\end{aligned}
$$

We have $c=d=c * d$. Thus, neut $\left(a^{n}\right)$ is unique and neut $\left(a^{n}\right) * \operatorname{neut}\left(a^{n}\right)=\operatorname{neut}\left(a^{n}\right)$.

Proposition 3. Let $(N, *)$ be a GAG-NET-Loop, $a \in N$ and $\left(a\right.$, neut $\left(a^{n}\right)$, anti $\left.\left(a^{n}\right)\right)$ is a generalized neutrosophic extended triplet with degree $n$. Then,

(1) $\left(a^{n} * a^{n}\right) * a^{n}=a^{n} *\left(a^{n} * a^{n}\right)$.

(2) neut $\left(a^{n}\right) * x=\operatorname{neut}\left(a^{n}\right) * y$, for any $x, y \in\left\{\operatorname{anti}\left(a^{n}\right)\right\}$.

(3) neut $\left(\right.$ neut $\left.\left(a^{n}\right)\right)=\operatorname{neut}\left(a^{n}\right)$.

(4) $a^{n} *\left(x *\right.$ neut $\left.\left(a^{n}\right)\right)=\left(x *\right.$ neut $\left.\left(a^{n}\right)\right) * a^{n}=\operatorname{neut}\left(a^{n}\right)$, for any $x \in\left\{\operatorname{anti}\left(a^{n}\right)\right\}$.

(5) $a^{n} *\left(\right.$ neut $\left.\left(a^{n}\right) * x\right)=\left(\right.$ neut $\left.\left(a^{n}\right) * x\right) * a^{n}=\operatorname{neut}\left(a^{n}\right)$, for any $x \in\left\{\operatorname{anti}\left(a^{n}\right)\right\}$.

(6) $\left(\right.$ neut $\left.\left(a^{n}\right) * x\right) *$ neut $\left(a^{n}\right)=\operatorname{neut}\left(a^{n}\right) *\left(\right.$ neut $\left.\left(a^{n}\right) * x\right)=\operatorname{neut}\left(a^{n}\right) * x$, for any $x \in\left\{\operatorname{anti}\left(a^{n}\right)\right\}$.

(7) neut $\left(\right.$ neut $\left.\left(a^{n}\right) * x\right) * \operatorname{anti}\left(\right.$ neut $\left.\left(a^{n}\right) * x\right)=a^{n}$, for any $x \in\left\{\operatorname{anti}\left(a^{n}\right)\right\}$.

\section{Proof.}

(1) For $a \in N$, neut $\left(a^{n}\right) * a^{n}=a^{n} * \operatorname{neut}\left(a^{n}\right)=a^{n}$, we have

$$
\left(a^{n} * a^{n}\right) * a^{n}=\left(a^{n} * a^{n}\right) *\left(\text { neut }\left(a^{n}\right) * a^{n}\right)=\left(a^{n} * \operatorname{neut}\left(a^{n}\right)\right) *\left(a^{n} * a^{n}\right)=a^{n} *\left(a^{n} * a^{n}\right) .
$$

(2) For any $x, y \in\left\{\operatorname{anti}\left(a^{n}\right)\right\}$, we have neut $\left(a^{n}\right) * x=\left(y * a^{n}\right) * x=\left(x * a^{n}\right) * y=\operatorname{neut}\left(a^{n}\right) * y$.

(3) From Proposition 2, we have neut $\left(a^{n}\right)$ exists neutral element and opposite element. For any $x \in\left\{\operatorname{anti}\left(a^{n}\right)\right\}$ and $y \in\left\{\operatorname{anti}\left(\right.\right.$ neut $\left.\left.\left(a^{n}\right)\right)\right\}$, 


$$
(y * x) * a^{n}=\left(a^{n} * x\right) * y=\operatorname{neut}\left(a^{n}\right) * y=\operatorname{neut}\left(\operatorname{neut}\left(a^{n}\right)\right) .
$$

Moreover,

$$
\begin{aligned}
\left((y * x) * a^{n}\right) * \operatorname{neut}\left(a^{n}\right) & =\left(\operatorname{neut}\left(a^{n}\right) * y\right) * \operatorname{neut}\left(a^{n}\right) \\
& =\left(y * \operatorname{neut}\left(a^{n}\right)\right) * \operatorname{neut}\left(a^{n}\right) \\
& =\left(\operatorname{neut}\left(a^{n}\right) * \operatorname{neut}\left(a^{n}\right)\right) * y \\
& =\operatorname{neut}\left(a^{n}\right) * y \\
& =\operatorname{neut}\left(\operatorname{neut}\left(a^{n}\right)\right) .
\end{aligned}
$$

Thus, neut $\left(a^{n}\right)=\operatorname{neut}\left(\operatorname{neut}\left(a^{n}\right)\right) * \operatorname{neut}\left(a^{n}\right)=\left((y * x) * a^{n}\right) * \operatorname{neut}\left(a^{n}\right)=\operatorname{neut}\left(\operatorname{neut}\left(a^{n}\right)\right)$.

(4) For any $x \in\left\{\operatorname{anti}\left(a^{n}\right)\right\}$, from Definition 11 and Proposition 2, we have

$$
\begin{aligned}
a^{n} *\left(x * \operatorname{neut}\left(a^{n}\right)\right) & =\left(a^{n} * \operatorname{neut}\left(a^{n}\right)\right) *\left(x * \operatorname{neut}\left(a^{n}\right)\right) \\
& =\left(a^{n} * x\right) *\left(\operatorname{neut}\left(a^{n}\right) * \operatorname{neut}\left(a^{n}\right)\right) \\
& =\operatorname{neut}\left(a^{n}\right) * \operatorname{neut}\left(a^{n}\right) \\
& =\operatorname{neut}\left(a^{n}\right),
\end{aligned}
$$

Thus, $a^{n} *\left(x * \operatorname{neut}\left(a^{n}\right)\right)=\left(x * \operatorname{neut}\left(a^{n}\right)\right) * a^{n}=\operatorname{neut}\left(a^{n}\right)$, for any $x \in\left\{\operatorname{anti}\left(a^{n}\right)\right\}$.

(5) For any $x \in\left\{\operatorname{anti}\left(a^{n}\right)\right\}$, we have

$$
\begin{aligned}
\left(\operatorname{neut}\left(a^{n}\right) * x\right) * a^{n} & =\left(\operatorname{neut}\left(a^{n}\right) * x\right) *\left(\operatorname{neut}\left(a^{n}\right) * a^{n}\right) \\
& =\left(\operatorname{neut}\left(a^{n}\right) * \operatorname{neut}\left(a^{n}\right)\right) *\left(x * a^{n}\right) \\
& =\operatorname{neut}\left(a^{n}\right) * \operatorname{neut}\left(a^{n}\right) \\
& =\operatorname{neut}\left(a^{n}\right), \\
a^{n} *\left(\operatorname{neut}\left(a^{n}\right) * x\right) & =\left(\operatorname{neut}\left(a^{n}\right) * a^{n}\right) *\left(\operatorname{neut}\left(a^{n}\right) * x\right) \\
& =\left(\operatorname{neut}\left(a^{n}\right) * \operatorname{neut}\left(a^{n}\right)\right) *\left(a^{n} * x\right) \\
& =\operatorname{neut}\left(a^{n}\right) * \operatorname{neut}\left(a^{n}\right) \\
& =\operatorname{neut}\left(a^{n}\right) .
\end{aligned}
$$

Thus, $a^{n} *\left(\operatorname{neut}\left(a^{n}\right) * x\right)=\left(\operatorname{neut}\left(a^{n}\right) * x\right) * a^{n}=\operatorname{neut}\left(a^{n}\right)$.

(6) For any $x \in\left\{\operatorname{anti}\left(a^{n}\right)\right\}$, we have

$$
\begin{aligned}
\left(\operatorname{neut}\left(a^{n}\right) * x\right) * \operatorname{neut}\left(a^{n}\right) & =\left(\operatorname{neut}\left(a^{n}\right) * x\right) *\left(a^{n} * x\right) \\
& =\left(\operatorname{neut}\left(a^{n}\right) * a^{n}\right) *(x * x) \\
& =\left(a^{n} * \operatorname{neut}\left(a^{n}\right)\right) *(x * x) \\
& \left.=\left(a^{n} * x\right) * \operatorname{neut}\left(a^{n}\right) * x\right) \\
& =\operatorname{neut}\left(a^{n}\right) *\left(\operatorname{neut}\left(a^{n}\right) * x\right), \\
\operatorname{neut}\left(a^{n}\right) *\left(\operatorname{neut}\left(a^{n}\right) * x\right) & =\left(x * a^{n}\right) *\left(\operatorname{neut}\left(a^{n}\right) * x\right) \\
& =\left(x * \operatorname{neut}\left(a^{n}\right)\right) *\left(a^{n} * x\right) \\
& =\left(x * \operatorname{neut}\left(a^{n}\right)\right) * \operatorname{neut}\left(a^{n}\right) \\
& =\left(\operatorname{neut}\left(a^{n}\right) * \operatorname{neut}\left(a^{n}\right)\right) * x \\
& =\operatorname{neut}\left(a^{n}\right) * x .
\end{aligned}
$$


Thus, $\left(\right.$ neut $\left.\left(a^{n}\right) * x\right) * \operatorname{neut}\left(a^{n}\right)=\operatorname{neut}\left(a^{n}\right) *\left(\operatorname{neut}\left(a^{n}\right) * x\right)=\operatorname{neut}\left(a^{n}\right) * x$.

(7) From (5) and (6), we have neut $\left(\right.$ neut $\left.\left(a^{n}\right) * x\right)=\operatorname{neut}\left(a^{n}\right), a^{n} \in \operatorname{anti}\left\{n e u t\left(a^{n}\right) * x\right\}$. From (2), neut $\left(a^{n}\right) * \operatorname{anti}\left(a^{n}\right)$ is unique, we have

$$
\operatorname{neut}\left(\text { neut }\left(a^{n}\right) * x\right) * \operatorname{anti}\left(\text { neut }\left(a^{n}\right) * x\right)=\operatorname{neut}\left(\operatorname{neut}\left(a^{n}\right) * x\right) * a^{n}=\operatorname{neut}\left(a^{n}\right) * a^{n}=a^{n} .
$$

Example 8. Let $S=\{a, b, c, d\}$, an operation $*$ on $S$ is defined as in Table 7. Since neut $(a)=a,\{$ anti $(a)\}=$ $\{a, b, c\}$, neut $(d)=a$, anti $(d)=d$ and $b^{2}=a, c^{2}=a$, so $(S, *)$ is a GAG-NET-Loop. We can get that (Corresponding to the results of Proposition 3):

Table 7. A GAG-NET-Loop of Example 8.

\begin{tabular}{lllll}
\hline$*$ & $\boldsymbol{a}$ & $\boldsymbol{b}$ & $\boldsymbol{c}$ & $\boldsymbol{d}$ \\
\hline$a$ & $a$ & $a$ & $a$ & $d$ \\
$b$ & $a$ & $a$ & $c$ & $d$ \\
$c$ & $a$ & $b$ & $a$ & $d$ \\
$d$ & $d$ & $d$ & $d$ & $a$ \\
\hline
\end{tabular}

(1) Being $\left(b^{2} * b^{2}\right) * b^{2}=b^{2} *\left(b^{2} * b^{2}\right),\left(d^{1} * d^{1}\right) * d^{1}=d^{1} *\left(d^{1} * d^{1}\right)$, that is $\left(a^{n} * a^{n}\right) * a^{n}=a^{n} *\left(a^{n} * a^{n}\right)$.

(2) Being $a * a=a * b=a * c$, that is for any $x, y \in\left\{\operatorname{anti}\left(c^{2}\right)\right\}$, neut $\left(c^{2}\right) * x=\operatorname{neut}\left(c^{2}\right) * y$.

(3) Being neut $\left(\right.$ neut $\left.\left(a^{1}\right)\right)=\operatorname{neut}\left(a^{1}\right)=a$, neut $\left(\right.$ neut $\left.\left(d^{1}\right)\right)=\operatorname{neut}\left(d^{1}\right)=a$, neut $\left(\right.$ neut $\left.\left(b^{2}\right)\right)=$ $\operatorname{neut}\left(b^{2}\right)=a, \operatorname{neut}\left(\operatorname{neut}\left(c^{2}\right)\right)=\operatorname{neut}\left(c^{2}\right)=a$, that is neut $\left(\operatorname{neut}\left(a^{n}\right)\right)=\operatorname{neut}\left(a^{n}\right)$.

(4) Being $c^{2} *\left(a *\right.$ neut $\left.\left(c^{2}\right)\right)=a,\left(a *\right.$ neut $\left.\left(c^{2}\right)\right) * c^{2}=a=\operatorname{neut}\left(c^{2}\right), c^{2} *\left(b * \operatorname{neut}\left(c^{2}\right)\right)=a,(b *$ neut $\left.\left(c^{2}\right)\right) * c^{2}=a=\operatorname{neut}\left(c^{2}\right), c^{2} *\left(c *\right.$ neut $\left.\left(c^{2}\right)\right)=a,\left(c *\right.$ neut $\left.\left(c^{2}\right)\right) * c^{2}=a=$ neut $\left(c^{2}\right)$, that is $c^{2} *$ $\left(x *\right.$ neut $\left.\left(c^{2}\right)\right)=\left(x *\right.$ neut $\left.\left(c^{2}\right)\right) * c^{2}=$ neut $\left(c^{2}\right)$, for any $x \in\left\{\operatorname{anti}\left(c^{2}\right)\right\}$. Being $d^{1} *\left(d *\right.$ neut $\left.\left(d^{1}\right)\right)=$ $a,\left(d *\right.$ neut $\left.\left(d^{1}\right)\right) * d^{1}=a=$ neut $\left(d^{1}\right)$, that is $d^{1} *\left(x *\right.$ neut $\left.\left(d^{1}\right)\right)=\left(x *\right.$ neut $\left.\left(d^{1}\right)\right) * d^{1}=\operatorname{neut}\left(d^{1}\right)$, for any $x \in\left\{\operatorname{anti}\left(d^{1}\right)\right\}$.

(5) Being $c^{2} *\left(\right.$ neut $\left.\left(c^{2}\right) * a\right)=a,\left(\right.$ neut $\left.\left(c^{2}\right) * a\right) * c^{2}=a=\operatorname{neut}\left(c^{2}\right), c^{2} *\left(\operatorname{neut}\left(c^{2}\right) * b\right)=a,\left(\right.$ neut $\left(c^{2}\right) *$ $b) * c^{2}=a=\operatorname{neut}\left(c^{2}\right), c^{2} *\left(\operatorname{neut}\left(c^{2}\right) * c\right)=a,\left(\operatorname{neut}\left(c^{2}\right) * c\right) * c^{2}=a=\operatorname{neut}\left(c^{2}\right)$, that is $c^{2} *$ $\left(\right.$ neut $\left.\left(c^{2}\right) * x\right)=\left(\right.$ neut $\left.\left(c^{2}\right) * x\right) * c^{2}=$ neut $\left(c^{2}\right)$, for any $x \in\left\{\operatorname{anti}\left(c^{2}\right)\right\}$. Being $d^{1} *\left(\right.$ neut $\left.\left(d^{1}\right) * d\right)=$ $a,\left(\operatorname{neut}\left(d^{1}\right) * d\right) * d^{1}=a=\operatorname{neut}\left(d^{1}\right)$, that is $d^{1} *\left(\operatorname{neut}\left(d^{1}\right) * x\right)=\left(\operatorname{neut}\left(d^{1}\right) * x\right) * d^{1}=\operatorname{neut}\left(d^{1}\right)$, for any $x \in\left\{\operatorname{anti}\left(d^{1}\right)\right\}$.

(6) Being neut $\left(c^{2}\right) * a=a,\left(\right.$ neut $\left.\left(c^{2}\right) * a\right) *$ neut $\left(c^{2}\right)=a$, neut $\left(c^{2}\right) *\left(\right.$ neut $\left.\left(c^{2}\right) * a\right)=a$; neut $\left(c^{2}\right) * b=a$, $\left(\right.$ neut $\left.\left(c^{2}\right) * b\right) *$ neut $\left(c^{2}\right)=a$, neut $\left(c^{2}\right) *\left(\right.$ neut $\left.\left(c^{2}\right) * b\right)=a ; \operatorname{neut}\left(c^{2}\right) * c=a,\left(\right.$ neut $\left.\left(c^{2}\right) * c\right) *$ neut $\left(c^{2}\right)=a$, neut $\left(c^{2}\right) *\left(\operatorname{neut}\left(c^{2}\right) * a\right)=a$; that is $\left(\right.$ neut $\left.\left(c^{2}\right) * x\right) *$ neut $\left(c^{2}\right)=$ neut $\left(c^{2}\right) *\left(\right.$ neut $\left(c^{2}\right) *$ $x)=\operatorname{neut}\left(c^{2}\right) * x$, for any $x \in\left\{\operatorname{anti}\left(c^{2}\right)\right\}$. Being neut $\left(d^{1}\right) * d=d,\left(\right.$ neut $\left.\left(d^{1}\right) * d\right) *$ neut $\left(d^{1}\right)=d$, neut $\left(d^{1}\right) *\left(\right.$ neut $\left.\left(d^{1}\right) * d\right)=d$, that is $\left(\right.$ neut $\left.\left(d^{1}\right) * x\right) *$ neut $\left(d^{1}\right)=\operatorname{neut}\left(d^{1}\right) *\left(\right.$ neut $\left.\left(d^{1}\right) * x\right)=$ neut $\left(d^{1}\right) * x$, for any $x \in\left\{\operatorname{anti}\left(d^{1}\right)\right\}$.

(7) Being neut $\left(\right.$ neut $\left.\left(c^{2}\right) * a\right) * \operatorname{anti}\left(\right.$ neut $\left.\left(c^{2}\right) * a\right)=a=c^{2} ;$ neut $\left(\right.$ neut $\left.\left(c^{2}\right) * b\right) * \operatorname{anti}\left(\right.$ neut $\left.\left(c^{2}\right) * b\right)=$ $a=c^{2} ;$ neut $\left(\right.$ neut $\left.\left(c^{2}\right) * c\right) * \operatorname{anti}\left(\right.$ neut $\left.\left(c^{2}\right) * c\right)=a=c^{2}$; that is neut $\left(\right.$ neut $\left.\left(c^{2}\right) * x\right) * \operatorname{anti}\left(\right.$ neut $\left(c^{2}\right) *$ $x)=c^{2}$, for any $x \in\left\{\operatorname{anti}\left(c^{2}\right)\right\}$. Being neut $\left(\right.$ neut $\left.\left(d^{1}\right) * d\right) * \operatorname{anti}\left(\right.$ neut $\left.\left(d^{1}\right) * d\right)=d^{1}$, that is neut $\left(\right.$ neut $\left.\left(d^{1}\right) * x\right) * \operatorname{anti}\left(\right.$ neut $\left.\left(d^{1}\right) * x\right)=d^{1}$, for any $x \in\left\{\operatorname{anti}\left(d^{1}\right)\right\}$.

Proposition 4. Let $(N, *)$ be a GAG-NET-Loop, then $\forall a, b \in N$, there are two positive integers $n$ and $m$ such that the following hold:

(1) neut $\left(a^{n}\right) *$ neut $\left(b^{m}\right)=\operatorname{neut}\left(a^{n} * b^{m}\right)$.

(2) $\operatorname{anti}\left(a^{n}\right) * \operatorname{anti}\left(b^{m}\right) \in\left\{\operatorname{anti}\left(a^{n} * b^{m}\right)\right\}$. 
Proof. Being $(N, *)$ be a GAG-NET-Loop, then for $a \in N$, there is a positive integer $n$, such that $a^{n}$ has the neutral element and opposite element, denoted by neut $\left(a^{n}\right)$ and $\operatorname{anti}\left(a^{n}\right)$, respectively. For $b \in N$, there is a positive integer $m$, such that $b^{m}$ has the neutral element and opposite element, denoted by $n e u t\left(b^{m}\right)$ and $\operatorname{anti}\left(b^{m}\right)$, respectively. Thus,

$$
\begin{aligned}
\left(\operatorname{neut}\left(a^{n}\right) * \operatorname{neut}\left(b^{m}\right)\right) *\left(a^{n} * b^{m}\right) & =\left(\operatorname{neut}\left(a^{n}\right) * a^{n}\right) *\left(\operatorname{neut}\left(b^{m}\right) * b^{m}\right) \\
& =a^{n} * b^{m} .
\end{aligned}
$$

In the same way, we have $\left(a^{n} * b^{m}\right) *\left(\right.$ neut $\left(a^{n}\right) *$ neut $\left.\left(b^{m}\right)\right)=a^{n} * b^{m}$.

That is,

$$
\left(a^{n} * b^{m}\right) *\left(\operatorname{neut}\left(a^{n}\right) * \operatorname{neut}\left(b^{m}\right)\right)=\left(\operatorname{neut}\left(a^{n}\right) * \operatorname{neut}\left(b^{m}\right)\right) *\left(a^{n} * b^{m}\right)=a^{n} * b^{m} .
$$

Moreover, for any $\operatorname{anti}\left(a^{n}\right) \in\left\{\operatorname{anti}\left(a^{n}\right)\right\}$ and $\operatorname{anti}\left(b^{m}\right) \in\left\{\operatorname{anti}\left(b^{m}\right)\right\}$, we can get

$$
\begin{aligned}
\left(\operatorname{anti}\left(a^{n}\right) * \operatorname{anti}\left(b^{m}\right)\right) *\left(a^{n} * b^{m}\right) & =\left(\operatorname{anti}\left(a^{n}\right) * a^{n}\right) *\left(\operatorname{anti}\left(b^{m}\right) * b^{m}\right) \\
& =\operatorname{neut}\left(a^{n}\right) * \operatorname{neut}\left(b^{m}\right) .
\end{aligned}
$$

Similarly, we have $\left(a^{n} * b^{m}\right) *\left(\operatorname{anti}\left(a^{n}\right) * \operatorname{anti}\left(b^{m}\right)\right)=\operatorname{neut}\left(a^{n}\right) * n e u t\left(b^{m}\right)$. That is:

$$
\left(a^{n} * b^{m}\right) *\left(\operatorname{anti}\left(a^{n}\right) * \operatorname{anti}\left(b^{m}\right)\right)=\left(\operatorname{anti}\left(a^{n}\right) * \operatorname{anti}\left(b^{m}\right)\right) *\left(a^{n} * b^{m}\right)=\operatorname{neut}\left(a^{n}\right) * \operatorname{neut}\left(b^{m}\right) .
$$

Thus, we have

$$
\operatorname{neut}\left(a^{n}\right) * \operatorname{neut}\left(b^{m}\right) \in\left\{\operatorname{neut}\left(a^{n} * b^{m}\right)\right\} .
$$

From this, by Proposition 2, we get neut $\left(a^{n}\right) *$ neut $\left(b^{m}\right)=$ neut $\left(a^{n} * b^{m}\right)$. Therefore, we get anti $\left(a^{n}\right) *$ $\operatorname{anti}\left(b^{m}\right) \in\left\{\operatorname{anti}\left(a^{n} * b^{m}\right)\right\}$.

Example 9. Apply the $(S, *)$ in Example 8, since neut $(a)=a,\{\operatorname{anti}(a)\}=\{a, b, c\}, \operatorname{neut}(d)=$ $a$, anti $(d)=d$ and $b^{2}=a, c^{2}=a$, so $(S, *)$ is a GAG-NET-Loop, we can get:

(1) Being neut $\left(c^{2}\right) * \operatorname{neut}\left(d^{1}\right)=a$, neut $\left(c^{2} * d^{1}\right)=a$, that is neut $\left(c^{2}\right) * \operatorname{neut}\left(d^{1}\right)=\operatorname{neut}\left(c^{2} * d^{1}\right)$.

(2) Being $a * d=b * d=c * d=d$, that is anti $\left(c^{2}\right) * \operatorname{anti}\left(d^{1}\right) \in\left\{\operatorname{anti}\left(c^{2} * d^{1}\right)\right\}$

Theorem 7. Let $(N, *)$ be a GAG-NET-Loop. Then, $N$ is a quasi regular AG-groupoid.

Proof. For any $a$ in $N$, by Definition 11 we have $\left(a^{n} * \operatorname{anti}\left(a^{n}\right)\right) * a^{n}=\operatorname{neut}\left(a^{n}\right) * a^{n}=a^{n}$. From this and Definition 4, we know that $N$ is a quasi regular AG-groupoid.

The following example shows that a quasi regular AG-groupoid may not be a GAG-NET-loop.

Example 10. Apply the $(S, *)$ in Example 1, Being $1=(1 * 3) * 1,2=(2 * 4) * 2,3=(3 * 1) * 3,4=$ $(4 * 2) * 4$, From Definition $4, S$ is a quasi regular AG-groupoid. However, it is not a GAG-NET-Loop.

Theorem 8. Let $(N, *)$ be a GAG-NET-Loop. Then, $N$ is a quasi fully regular AG-groupoid.

Proof. Suppose $a \in N$ and $\left(a, n e u t\left(a^{n}\right)\right.$, anti $\left.\left(a^{n}\right)\right)$ is a generalized neutrosophic extended triplet with degree $n$, then there exists $m \in\left\{\operatorname{anti}\left(a^{n}\right)\right\}, a^{n} * m=m * a^{n}=\operatorname{neut}\left(a^{n}\right)$. Denote $p=m * \operatorname{neut}\left(a^{n}\right), q=$ $\operatorname{neut}\left(a^{n}\right) ; r=m, s=\operatorname{neut}\left(a^{n}\right) ; t=m, u=\operatorname{neut}\left(a^{n}\right) ; v=m ; w=m * \operatorname{neut}\left(a^{n}\right) ; x=m, y=\operatorname{neut}\left(a^{n}\right)$, then 


$$
\begin{aligned}
& \left(p *\left(a^{n}\right)^{2}\right) * q=\left(\left(m * \operatorname{neut}\left(a^{n}\right)\right) *\left(a^{n}\right)^{2}\right) * \operatorname{neut}\left(a^{n}\right) \\
& =\left(\left(\left(a^{n}\right)^{2} * \operatorname{neut}\left(a^{n}\right)\right) * m\right) * \operatorname{neut}\left(a^{n}\right) \quad(\text { by the left invertive law }) \\
& =\left(\left(\left(a^{n} * a^{n}\right) * \operatorname{neut}\left(a^{n}\right)\right) * m\right) * \operatorname{neut}\left(a^{n}\right) \\
& =\left(\left(\left(\text { neut }\left(a^{n}\right) * a^{n}\right) * a^{n}\right) * m\right) * \text { neut }\left(a^{n}\right) \quad \text { (by the left invertive law) } \\
& =\left(\left(a^{n} * a^{n}\right) * m\right) * \operatorname{neut}\left(a^{n}\right) \\
& \left.=\left(\left(m * a^{n}\right) * a^{n}\right) * \operatorname{neut}\left(a^{n}\right) \quad \text { (by the left invertive law }\right) \\
& =\left(\operatorname{neut}\left(a^{n}\right) * a^{n}\right) * \operatorname{neut}\left(a^{n}\right) \\
& =a^{n} * \operatorname{neut}\left(a^{n}\right)=a^{n} \text {, } \\
& \left(r * a^{n}\right) *\left(a^{n} * s\right)=\left(m * a^{n}\right) *\left(a^{n} * \operatorname{neut}\left(a^{n}\right)\right)=\operatorname{neut}\left(a^{n}\right) * a^{n}=a^{n}, \\
& \left(a^{n} * t\right) *\left(a^{n} * u\right)=\left(a^{n} * m\right) *\left(a^{n} * \operatorname{neut}\left(a^{n}\right)\right)=\operatorname{neut}\left(a^{n}\right) * a^{n}=a^{n}, \\
& \left(a^{n} * a^{n}\right) * v=\left(a^{n} * a^{n}\right) * m=\left(m * a^{n}\right) * a^{n}=\operatorname{neut}\left(a^{n}\right) * a^{n}=a^{n}, \\
& w *\left(a^{n} * a^{n}\right)=\left(m * \operatorname{neut}\left(a^{n}\right)\right) *\left(a^{n} * a^{n}\right) \\
& =\left(m * a^{n}\right) *\left(\text { neut }\left(a^{n}\right) * a^{n}\right) \quad \text { (by the medial law) } \\
& =\left(m * a^{n}\right) * a^{n} \\
& =\operatorname{neut}\left(a^{n}\right) * a^{n}=a^{n}, \\
& \left(x * a^{n}\right) *\left(y * a^{n}\right)=\left(m * a^{n}\right) *\left(\operatorname{neut}\left(a^{n}\right) * a^{n}\right)=\operatorname{neut}\left(a^{n}\right) * a^{n}=a^{n} .
\end{aligned}
$$

Moreover, from Proposition 4, we get:

$$
\operatorname{neut}\left(a^{n}\right) * \operatorname{neut}\left(b^{m}\right)=\operatorname{neut}\left(a^{n} * b^{m}\right), \operatorname{anti}\left(a^{n}\right) * \operatorname{anti}\left(b^{m}\right) \in\left\{\operatorname{anti}\left(a^{n} * b^{m}\right)\right\} .
$$

If $b^{m}=a^{n}$, we have neut $\left(a^{n}\right) * \operatorname{neut}\left(a^{n}\right)=\operatorname{neut}\left(a^{n} * a^{n}\right), \operatorname{anti}\left(a^{n}\right) * \operatorname{anti}\left(a^{n}\right) \in\left\{\operatorname{anti}\left(a^{n} * a^{n}\right)\right\}$, there exists $k \in\left\{\operatorname{anti}\left(a^{n} * a^{n}\right)\right\}$. Denote $z=k * m$, then

$$
\begin{aligned}
\left(\left(a^{n}\right)^{2} * z\right) *\left(a^{n}\right)^{2} & =\left(\left(a^{n} * a^{n}\right) * z\right) *\left(a^{n}\right)^{2} \\
& \left.=\left(\left(z * a^{n}\right) * a^{n}\right) *\left(a^{n}\right)^{2} \quad \text { (applying the left invertive law }\right) \\
& =\left(\left(a^{n}\right)^{2} * a^{n}\right) *\left(z * a^{n}\right) \quad(\text { applying the left invertive law }) \\
& =\left(\left(a^{n}\right)^{2} * a^{n}\right) *\left((k * m) * a^{n}\right) \\
& =\left(\left(a^{n}\right)^{2} * a^{n}\right) *\left(\left(a^{n} * m\right) * k\right) \quad(\text { by the left invertive law }) \\
& =\left(\left(a^{n}\right)^{2} * a^{n}\right) *\left(\text { neut }\left(a^{n}\right) * k\right) \quad\left(\text { by } m \in\left\{\text { anti }\left(a^{n}\right)\right\}\right) \\
& =\left(\left(a^{n} * a^{n}\right) *\left(\text { neut }\left(a^{n}\right) * a^{n}\right)\right) *\left(\text { neut }\left(a^{n}\right) * k\right) \\
& =\left(\left(a^{n} * \text { neut }\left(a^{n}\right)\right) *\left(a^{n} * a^{n}\right)\right) *\left(\text { neut }\left(a^{n}\right) * k\right) \quad(\text { applying the medial law }) \\
& =\left(a^{n} *\left(a^{n}\right)^{2}\right) *\left(\text { neut }\left(a^{n}\right) * k\right) \\
& =\left(a^{n} * \text { neut }\left(a^{n}\right)\right) *\left(\left(a^{n}\right)^{2} * k\right) \quad(\text { applying the medial law }) \\
& =a^{n} * \text { neut }\left(a^{n} * a^{n}\right) \quad\left(\text { by the definition of } k \in\left\{\text { anti }\left(a^{n} * a^{n}\right)\right\}\right) \\
& =a^{n} *\left(\text { neut }\left(a^{n}\right) * n e u t\left(a^{n}\right)\right) \\
& =a^{n} * \text { neut }\left(a^{n}\right) \quad(\text { by Proposition } 2(2)) \\
& =a^{n} .
\end{aligned}
$$

Therefore, combining above results, by Definition 5, we know that $N$ is a quasi fully regular AG-groupoid. 
The following example shows that a quasi fully regular AG-groupoid may not be a GAG-NET-loop.

Example 11. Applying the $(S, *)$ in Example 1, when $a=1, p=1, q=3, r=4, s=3, t=2, u=3, v=$ $2, w=2, x=4, y=2, z=3$, we have $a^{2}=2$, and

$$
\begin{aligned}
1 & =(1 * 2) * 3=(4 * 1) *(1 * 3)=(1 * 2) *(1 * 3) \\
& =(1 * 1) * 2=2 *(1 * 1)=(4 * 1) *(2 * 1) \\
& =(2 * 3) * 2
\end{aligned}
$$

When $a=4, p=1, q=3, r=4, s=4, t=3, u=2, v=3, w=3, x=4, y=4, z=2$, we have $a^{2}=3$, and

$$
\begin{aligned}
4 & =(1 * 3) * 3=(4 * 4) *(4 * 4)=(4 * 3) *(4 * 2) \\
& =(4 * 4) * 3=3 *(4 * 4)=(4 * 4) *(4 * 4) \\
& =(3 * 2) * 3
\end{aligned}
$$

Being $2^{2}=1,3^{3}=1$, from Definition $5, S$ is a quasi fully regular AG-groupoid. However, it is not a GAG-NET-Loop.

Definition 13. An AG-groupoid $(S, *)$ is called a quasi strong inverse AG-groupoid, if the following conditions are satisfied: for any $a \in S$, there exists a positive integer $n, a^{n} \in S$, and a unary operation $a^{n} \rightarrow\left(a^{n}\right)^{-1}$ on $S$ such that

$$
\left(\left(a^{n}\right)^{-1}\right)^{-1}=a^{n}, \quad\left(a^{n} *\left(a^{n}\right)^{-1}\right) * a^{n}=a^{n} *\left(a^{n} *\left(a^{n}\right)^{-1}\right)=a^{n}, a^{n} *\left(a^{n}\right)^{-1}=\left(a^{n}\right)^{-1} * a^{n} .
$$

Theorem 9. Let $(N, *)$ be a groupoid. Then, $N$ is a GAG-NET-Loop if and only if it is a quasi strong inverse AG-groupoid.

Proof. Necessity: Suppose $N$ is a GAG-NET-Loop, from Definition 12, for each $a \in N$, there exists a generalized neutrosophic extended triplet with degree $\mathrm{n}$ denoted by $\left(a, n e u t\left(a^{n}\right)\right.$, anti $\left.\left(a^{n}\right)\right)$. Set

$$
\left(a^{n}\right)^{-1}=\operatorname{neut}\left(a^{n}\right) * \operatorname{anti}\left(a^{n}\right),
$$

by Proposition 3(2), neut $\left(a^{n}\right) * \operatorname{anti}\left(a^{n}\right)$ is unique, so $\left(a^{n}\right)^{-1}$ is unique. By Proposition 3(7), we have

$$
\left(\left(a^{n}\right)^{-1}\right)^{-1}=\operatorname{neut}\left(\operatorname{neut}\left(a^{n}\right) * \operatorname{anti}\left(a^{n}\right)\right) * \operatorname{anti}\left(\operatorname{neut}\left(a^{n}\right) * \operatorname{anti}\left(a^{n}\right)\right)=a^{n} .
$$

Being

$$
\begin{aligned}
& \left(a^{n}\right)^{-1} * a^{n}=\left(\operatorname{neut}\left(a^{n}\right) * \operatorname{anti}\left(a^{n}\right)\right) * a^{n} \\
& \qquad \begin{aligned}
a^{n} *\left(a^{n}\right)^{-1} & =a^{n} *\left(\operatorname{neut}\left(a^{n}\right) * \operatorname{anti}\left(a^{n}\right)\right) \\
& =\left(\operatorname{neut}\left(a^{n}\right) * a^{n}\right) *\left(\operatorname{neut}\left(a^{n}\right) * \operatorname{anti}\left(a^{n}\right)\right) \\
& =\left(\operatorname{neut}\left(a^{n}\right) * \operatorname{neut}\left(a^{n}\right)\right) *\left(a^{n} * \operatorname{anti}\left(a^{n}\right)\right) \\
& =\operatorname{neut}\left(a^{n}\right),
\end{aligned}
\end{aligned}
$$

we have

$$
\begin{gathered}
\left(a^{n}\right)^{-1} * a^{n}=a^{n} *\left(a^{n}\right)^{-1}, \\
\left(a^{n} *\left(a^{n}\right)^{-1}\right) * a^{n}=\operatorname{neut}\left(a^{n}\right) * a^{n}=a^{n},
\end{gathered}
$$




$$
\begin{gathered}
a^{n} *\left(a^{n} *\left(a^{n}\right)^{-1}\right)=a^{n} * \operatorname{neut}\left(a^{n}\right)=a^{n}, \\
\left(a^{n} *\left(a^{n}\right)^{-1}\right) * a^{n}=a^{n} *\left(a^{n} *\left(a^{n}\right)^{-1}\right)=a^{n} .
\end{gathered}
$$

From Definition 13, $N$ is a quasi strong inverse AG-groupoid.

Sufficiency: If $N$ is a quasi strong inverse AG-groupoid, and $\left(a^{n}\right)^{-1} \in N$, such that $a^{n} *\left(a^{n}\right)^{-1}=$ $\left(a^{n}\right)^{-1} * a^{n}$ and $\left(a^{n} *\left(a^{n}\right)^{-1}\right) * a^{n}=a^{n} *\left(a^{n} *\left(a^{n}\right)^{-1}\right)=a^{n}$. Set

$$
\operatorname{neut}\left(a^{n}\right)=a^{n} *\left(a^{n}\right)^{-1},
$$

then $\operatorname{neut}\left(a^{n}\right) * a^{n}=\left(a^{n} *\left(a^{n}\right)^{-1}\right) * a^{n}=a^{n} *\left(a^{n} *\left(a^{n}\right)^{-1}\right)=a^{n} * \operatorname{neut}\left(a^{n}\right)=a^{n}$,

$$
a^{n} *\left(a^{n}\right)^{-1}=\left(a^{n}\right)^{-1} * a^{n}=\operatorname{neut}\left(a^{n}\right) .
$$

From Definition 12, we have that $N$ is a GAG-NET-Loop and $\left(a^{n}\right)^{-1} \in\left\{\operatorname{anti}\left(a^{n}\right)\right\}$.

Example 12. Applying $(S, *)$ in Example 8, we know that it is a GAG-NET-Loop. We will show that it is a quasi strong inverse AG-groupoid in the following.

For $d$, there exists an inverse element $d^{-1}=d$, such that $\left(d^{-1}\right)^{-1}=d,\left(d * d^{-1}\right) * d=d *\left(d * d^{-1}\right)=$ $d, d * d^{-1}=d^{-1} * d=a$, so $d$ is quasi strong inverse. $a$ is quasi strong inverse for the same reason. Moreover, being $b^{2}=a, c^{2}=a, b$ and $c$ are quasi strong inverse, thus $(S, *)$ is a quasi strong inverse AG-groupoid by Definition 13.

Definition 14. Let $(N, *)$ be a GAG-NET-Loop. $N$ is called a weak commutative GAG-NET-Loop if $\forall a, b \in N$, there exist a generalized neutrosophic extended triplet with degree $n$ (denoted by $\left(a\right.$, neut $\left(a^{n}\right)$, anti $\left.\left(a^{n}\right)\right)$ ) and a generalized neutrosophic extended triplet with degree $m$ (denoted by $\left(b, n e u t\left(b^{m}\right)\right.$, anti $\left.\left(b^{m}\right)\right)$ ), $n, m \in Z^{+}$, $a^{n} * \operatorname{neut}\left(b^{m}\right)=\operatorname{neut}\left(b^{m}\right) * a^{n}$.

Example 13. Let $S=\{1,2,3,4,5,6,7\}$, an operation $*$ on $S$ is defined as in Table 8. Since $(1,1,1),(2,2,2)$ and $(6,6,6)$ are neutrosophic extended triplets, but 3, 4, 5, 7 do not have the neutral element and opposite element, thus $S$ is not an AG-NET-Loop. Moreover $3^{2}=1,4^{2}=1,5^{2}=2,7^{2}=6$ have the neutral element and opposite element, so $(S, *)$ is a GAG-NET-Loop. It is not a commutative GAG-NET-Loop being $3 * 1 \neq 1 * 3$. We can show that it is a weak commutative GAG-NET-Loop.

For 1,2,3,4,5,6 and 7, there exist positive integers $1,1,2,2,2,1$ and 2 , respectively, thus $S^{\prime}=$ $\left\{1^{1}, 2^{1}, 3^{2}, 4^{2}, 5^{2}, 6^{1}, 7^{2}\right\}=\{1,2,6\}$ being $3^{2}=1,4^{2}=1,5^{2}=2,7^{2}=6$. We know that neut $(1)=$ 1, neut $(2)=2$, neut $(6)=6$, thus $\{$ neut $(1)$, neut $(2)$, neut $(6)\} \subseteq S^{\prime}$. In Table 8, we can get the sub algebra system $\left(S^{\prime}, *\right)$ of $(S, *)$ as in Table 9 , and $\left(S^{\prime}, *\right)$ is commutative. Thus, $(S, *)$ is a weak commutative GAG-NET-Loop.

Table 8. The operation table of Example 13.

\begin{tabular}{llllllll}
\hline$*$ & $\mathbf{1}$ & $\mathbf{2}$ & $\mathbf{3}$ & $\mathbf{4}$ & $\mathbf{5}$ & $\mathbf{6}$ & $\mathbf{7}$ \\
\hline 1 & 1 & 2 & 3 & 4 & 5 & 6 & 7 \\
2 & 2 & 2 & 2 & 2 & 2 & 2 & 2 \\
3 & 4 & 2 & 1 & 3 & 5 & 6 & 7 \\
4 & 3 & 2 & 4 & 1 & 5 & 6 & 7 \\
5 & 5 & 2 & 5 & 5 & 2 & 2 & 2 \\
6 & 6 & 2 & 6 & 6 & 2 & 6 & 6 \\
7 & 7 & 2 & 7 & 7 & 2 & 6 & 6 \\
\hline
\end{tabular}


Table 9. The sub algebra system $S^{\prime}$ of $S$ in Example 13.

\begin{tabular}{llll}
\hline$*$ & $\mathbf{1}$ & $\mathbf{2}$ & $\mathbf{6}$ \\
\hline 1 & 1 & 2 & 6 \\
2 & 2 & 2 & 2 \\
6 & 6 & 2 & 6 \\
\hline
\end{tabular}

Example 14. Let $S=\{1,2,3,4\}$, an operation $*$ on $S$ is defined as in Table 10. Being neut $(1) * 2=4 \neq 3=$ $2 *$ neut(1), $S$ is not a weak commutative GAG-NET-Loop. Moreover, it is not a commutative AG-NET-Loop.

Table 10. The operation table of Example 14.

\begin{tabular}{lllll}
\hline $\boldsymbol{*}$ & $\mathbf{1}$ & $\mathbf{2}$ & $\mathbf{3}$ & $\mathbf{4}$ \\
\hline 1 & 1 & 4 & 2 & 3 \\
2 & 3 & 2 & 4 & 1 \\
3 & 4 & 1 & 3 & 2 \\
4 & 2 & 3 & 1 & 4 \\
\hline
\end{tabular}

Proposition 5. Let $(N, *)$ be a GAG-NET-Loop. Then, $(N, *)$ is a weak commutative GAG-NET-Loop if and only if $N$ satisfies the following conditions: $\forall a, b \in N$, there exist a generalized neutrosophic extended triplet with degree $n$ (denoted by $\left(a\right.$, neut $\left(a^{n}\right)$, anti $\left.\left(a^{n}\right)\right)$ ) and a generalized neutrosophic extended triplet with degree $m$ (denoted by $\left(b, n e u t\left(b^{m}\right)\right.$, anti $\left.\left.\left(b^{m}\right)\right)\right), n, m \in Z^{+}, a^{n} * b^{m}=b^{m} * a^{n}$.

Proof. Necessity: If $(N, *)$ is a weak commutative GAG-NET-Loop, then there are two positive integers $n, m$, such that $a^{n}$ and $b^{m}$ have the neutral element and opposite element. Thus, from Definition 14 , $\forall a, b \in N$, we have

$$
\begin{aligned}
a^{n} * b^{m} & =\left(\operatorname{neut}\left(a^{n}\right) * a^{n}\right) *\left(b^{m} * \text { neut }\left(b^{m}\right)\right) \\
& =\left(\operatorname{neut}\left(a^{n}\right) * b^{m}\right) *\left(a^{n} * \operatorname{neut}\left(b^{m}\right)\right) \\
& =\left(b^{m} * \operatorname{neut}\left(a^{n}\right)\right) *\left(\operatorname{neut}\left(b^{m}\right) * a^{n}\right) \\
& =\left(b^{m} * \operatorname{neut}\left(b^{m}\right)\right) *\left(\operatorname{neut}\left(a^{n}\right) * a^{n}\right) \\
& =b^{m} * a^{n} .
\end{aligned}
$$

Sufficiency: If $(N, *)$ is a GAG-NET-Loop, then for $a \in N$, there is a positive integer $n$, such that $a^{n}$ has the neutral element and opposite element, denoted by neut $\left(a^{n}\right)$ and $a n t i\left(a^{n}\right)$, respectively. For $b \in N$, there is a positive integer $m$, such that $b^{m}$ has the neutral element and opposite element, denoted by neut $\left(b^{m}\right)$ and anti $\left(b^{m}\right)$, respectively. Suppose that $(N, *)$ satisfies the conditions $a^{n} * b^{m}=$ $b^{m} * a^{n}$, From Proposition 2, we have neut $\left(b^{m}\right)$ exists neutral element and opposite element. We get $a^{n} *$ neut $\left(b^{m}\right)=\operatorname{neut}\left(b^{m}\right) * a^{n}$. From Definition 14 , we know that $(N, *)$ is a weak commutative GAG-NET-Loop.

Definition 15. A GAG-NET-Loop $(S, *)$ is called a quasi Clifford AG-groupoid, if it is a quasi strong inverse $A G$-groupoid and for any $a, b \in S$, there are two positive integers $n, m$ such that

$$
a^{n} *\left(b^{m} *\left(b^{m}\right)^{-1}\right)=\left(b^{m} *\left(b^{m}\right)^{-1}\right) * a^{n} .
$$

Theorem 10. Let $(N, *)$ be a groupoid. Then, $N$ is a weak commutative GAG-NET-Loop if and only if it is a quasi Clifford AG-groupoid. 
Proof. Necessity: Suppose that $N$ is a weak commutative GAG-NET-Loop. By Theorem 9, we know that $N$ is a quasi strong inverse AG-groupoid, then $\forall a, b \in N$ there are two positive integers $n, m$, such that $a^{n}$ and $b^{m}$ have the neutral element and opposite element. Set

$$
\left(a^{n}\right)^{-1}=\operatorname{neut}\left(a^{n}\right) * \operatorname{anti}\left(a^{n}\right) .
$$

For any $a, b \in N$, we have

$$
a^{n} *\left(b^{m} *\left(b^{m}\right)^{-1}\right)=a^{n} * \operatorname{neut}\left(b^{m}\right)=\operatorname{neut}\left(b^{m}\right) * a^{n}=\left(b^{m} *\left(b^{m}\right)^{-1}\right) * a^{n} .
$$

From Definition 15, we know that $N$ is a quasi Clifford AG-groupoid.

Sufficiency: Assume that $N$ is a quasi Clifford AG-groupoid, from Definition 15, it is a quasi strong inverse AG-groupoid. By Theorem 9, we know that $N$ is a GAG-NET-Loop. Then, $\forall a, b \in N$ there are two positive integers $n, m$, such that $a^{n}$ and $b^{m}$ have the neutral element and opposite element, $\left(a^{n}\right)^{-1} \in N,\left(b^{m}\right)^{-1} \in N$. Set

$$
\operatorname{neut}\left(a^{n}\right)=a^{n} *\left(a^{n}\right)^{-1}, \operatorname{neut}\left(b^{m}\right)=b^{m} *\left(b^{m}\right)^{-1} .
$$

From Definition 15, being $a^{n} *\left(b^{m} *\left(b^{m}\right)^{-1}\right)=\left(b^{m} *\left(b^{m}\right)^{-1}\right) * a^{n}$, we have $a^{n} *$ neut $\left(b^{m}\right)=$ $n e u t\left(b^{m}\right) * a^{n}$. We can get that $N$ is a weak commutative GAG-NET-Loop by Definition 14 .

Example 15. Let $S=\{1,2,3,4,5,6,7,8\}$, an operation $*$ on $S$ is defined as in Table 11. It is a weak commutative GAG-NET-Loop. We show that it is a quasi Clifford AG-groupoid. From Theorem 9, we can see that $(S, *)$ is a quasi strong inverse AG-groupoid. We just show for any $x, y \in S$, there are two positive integers $n$ and $m$ such that $x^{n} *\left(y^{m} *\left(y^{m}\right)^{-1}\right)=\left(y^{m} *\left(y^{m}\right)^{-1}\right) * x^{n}$.

In Example 15, 1,2,3, 4, 5, 6,7 and 8, there exist positive integers 1, 1,2,2, 2,1,2 and 2, respectively, and set $1^{-1}=1,2^{-1}=2,\left(3^{2}\right)^{-1}=1,\left(4^{2}\right)^{-1}=1,\left(5^{2}\right)^{-1}=2,6^{-1}=6,\left(7^{2}\right)^{-1}=6,\left(8^{2}\right)^{-1}=6$. For any $x, y \in\left\{1^{1}, 2^{1}, 3^{2}, 4^{2}, 5^{2}, 6^{1}, 7^{2}, 8^{2}\right\}$, without losing generality, let $x=1, y=2$, we can get $1^{1} *\left(2^{1} *\left(2^{1}\right)^{-1}\right)=$ $\left(2^{1} *\left(2^{1}\right)^{-1}\right) * 1^{1}=2$. We can verify other cases, thus $(S, *)$ is a quasi Clifford AG-groupoid.

Table 11. The operation table of Example 15.

\begin{tabular}{lllllllll}
\hline $\boldsymbol{*}$ & $\mathbf{1}$ & $\mathbf{2}$ & $\mathbf{3}$ & $\mathbf{4}$ & $\mathbf{5}$ & $\mathbf{6}$ & $\mathbf{7}$ & $\mathbf{8}$ \\
\hline 1 & 1 & 2 & 3 & 4 & 5 & 6 & 7 & 8 \\
2 & 2 & 2 & 2 & 2 & 2 & 2 & 2 & 2 \\
3 & 4 & 2 & 1 & 3 & 5 & 6 & 7 & 8 \\
4 & 3 & 2 & 4 & 1 & 5 & 6 & 7 & 8 \\
5 & 5 & 2 & 5 & 5 & 2 & 2 & 2 & 2 \\
6 & 6 & 2 & 6 & 6 & 2 & 6 & 6 & 6 \\
7 & 7 & 2 & 7 & 7 & 2 & 6 & 6 & 6 \\
8 & 8 & 2 & 8 & 8 & 2 & 6 & 6 & 6 \\
\hline
\end{tabular}

Example 16. Let $S=\{1,2,3,4,5\}$, an operation $*$ on $S$ is defined as in Table 12. it is not a weak commutative GAG-NET-Loop. We show that there exist $x, y \in S$, for any two positive integers $n$ and $m$ such that $x^{n} *\left(y^{m} *\right.$ $\left.\left(y^{m}\right)^{-1}\right) \neq\left(y^{m} *\left(y^{m}\right)^{-1}\right) * x^{n}$.

In Example 16, for any $n, m \in Z^{+}, 1^{n}=1,2^{m}=2$ and $\left(1^{n}\right)^{-1}=1,\left(2^{m}\right)^{-1}=2$, but $1^{n} *\left(2^{m} *\right.$ $\left.\left(2^{m}\right)^{-1}\right)=4 \neq 3=\left(2^{m} *\left(2^{m}\right)^{-1}\right) * 1^{n}$. That is, for $1,2 \in S$, there are not two positive integers $n, m$ such that $1^{n} *\left(2^{m} *\left(2^{m}\right)^{-1}\right)=\left(2^{m} *\left(2^{m}\right)^{-1}\right) * 1^{n}$. Thus, $(S, *)$ is not a quasi Clifford AG-groupoid. 
Table 12. The operation table of Example 16.

\begin{tabular}{llllll}
\hline $\boldsymbol{*}$ & $\mathbf{1}$ & $\mathbf{2}$ & $\mathbf{3}$ & $\mathbf{4}$ & $\mathbf{5}$ \\
\hline 1 & 1 & 4 & 2 & 3 & 1 \\
2 & 3 & 2 & 4 & 1 & 3 \\
3 & 4 & 1 & 3 & 2 & 4 \\
4 & 2 & 3 & 1 & 4 & 2 \\
5 & 1 & 4 & 2 & 3 & 5 \\
\hline
\end{tabular}

\section{Conclusions}

We thoroughly study the GAG-NET-Loop from the perspective of the AG-groupoid theory and obtained some important results. Figures 1 and 2 give the relations of the GAG-NET-Loop and other algebraic structures.

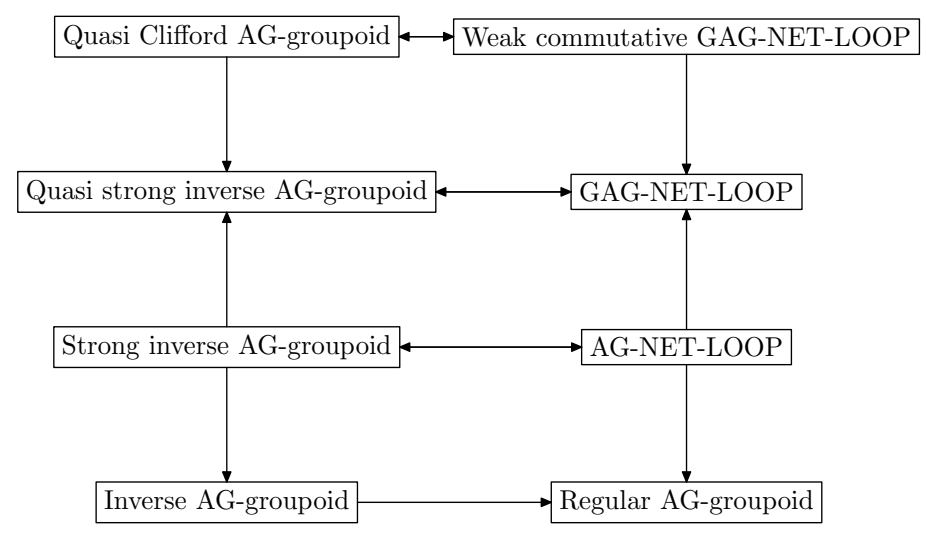

Figure 1. The relations of GAG-NET-Loop and other algebraic structures.

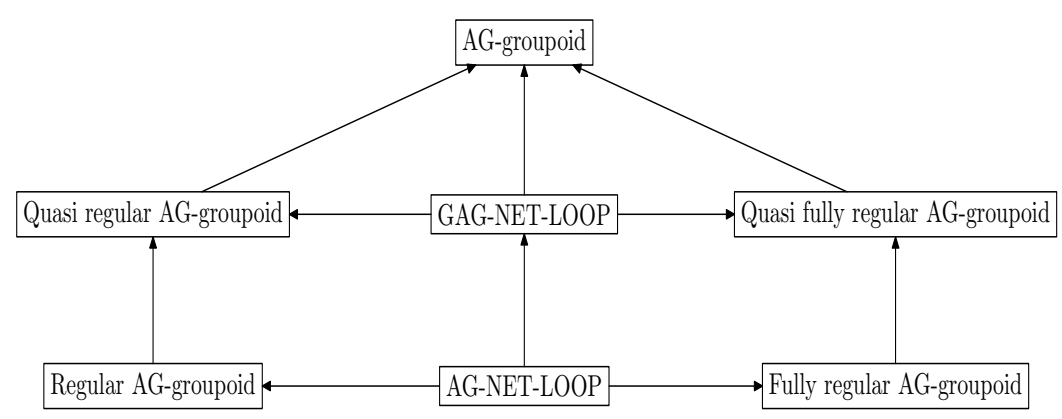

Figure 2. The relations of GAG-NET-Loop and other AG-groupoids.

As can be seen in Figure 1, we prove that the AG-NET-Loop is equal to the strong inverse AG-groupoid, the GAG-NET-Loop is equal to the quasi strong inverse AG-groupoid, and the weak commutative GAG-NET-Loop is equal to the quasi Clifford AG-groupoid.

As can be seen in Figure 2, we prove that a GAG-NET-loop is a quasi regular AG-groupoid, but a quasi regular AG-groupoid may not be a GAG-NET-loop; a GAG-NET-loop is a quasi fully regular AG-groupoid, but a quasi fully regular AG-groupoid may not be a GAG-NET-loop.

Figure 3 can be used to further express the relationships among GAG-NET-Loop and some algebraic systems. Here, as shown in Example 2, A represents a commutative AG-NET-Loop; as shown in Example 15, B represents a weak commutative GAG-NET-Loop, but it is not an AG-NET-Loop; as is shown in Example 14, C represents a non-commutative AG-NET-Loop; D represents a GAG-NET- Loop, but it is neither an AG-NET-Loop nor a weak commutative GAG-NET-Loop; as shown in Example 10, E represents a quasi regular AG-groupoid, but it is not a GAG-NET-Loop; and as shown in Example 11, F represents a quasi fully regular AG-groupoid, but it is not a GAG-NET-Loop. A+B represents a weak commutative GAG-NET-Loop, $A+C$ represents an AG-NET-Loop, $A+B+C+D$ represents a 
GAG-NET-Loop, $\mathrm{A}+\mathrm{B}+\mathrm{C}+\mathrm{D}+\mathrm{E}$ represents a quasi regular AG-groupoid, and $\mathrm{A}+\mathrm{B}+\mathrm{C}+\mathrm{D}+\mathrm{F}$ represents a quasi fully regular AG-groupoid.

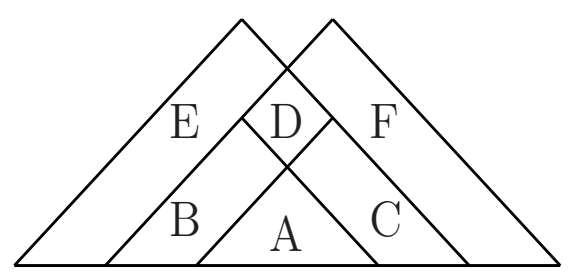

Figure 3. The relationships among some algebraic systems and GAG-NET-Loop.

All these results are interesting for the exploration of the structure characterization of GAG-NET-Loop. As the next research topics, we want to find some special GAG-NET-Loops which can be decomposed into some smaller GAG-NET-Loops, and explore the relationship between these special GAG-NET-Loops and the related AG-groupoids.

Author Contributions: Conceptualization, Funding acquisition, Writing_review and editing, X.Z.; Data curation, Software, Writing — original draft, Writing-review and editing, X.A.; Resources, Writing-review and editing, Y.M.

Funding: This research was funded by National Natural Science Foundation of China (Grant No. 61976130).

Conflicts of Interest: The authors declare no conflict of interest.

\section{References}

1. Kazim, M.; Naseeruddin, M. On almost semigroups. Aligarh Bull. Math. 1972, 2, 1-7.

2. Holgate, P. Groupoids satisfying a simple invertive law. Math. Stud. 1992, 61, 101-106.

3. Mushtaq, Q.; Iqbal, Q. Decomposition of a locally associative LA-semigroup. Semigroup Forum 1990, 41, 155-164. [CrossRef]

4. Proti, P.V.; Stevanovi, N. AG-test and some general properties of Abel-Grassmann's groupoids. Pure Math. Appl. 1995, 6, 371-383.

5. Khan, M.; Faisal; Amjad, V. On some classes of Abel-Grassmann's groupoids. J. Adv. Res. Pure Math. 2011, 3, 109-119. [CrossRef]

6. Faisal; Khan, A.; Davvaz, B. On fully regular AG-groupoids. Afr. Mat. 2014, 25, 449-459. [CrossRef]

7. Khan, M.; Anis, S. On semilattice decomposition of an Abel-Grassmann's groupoid. Acta Math. Sin. Engl. Ser. 2012, 28, 1461-1468. [CrossRef]

8. Rashad, M.; Ahmad, I.; Shah, M.; Khuhro, Z.U.A. Left transitive AG-groupoids. Mathematics 2014, $46,547-552$.

9. Iqbal, M.; Ahmad, I. Ideals in CA-AG-groupoids. Indian J. Pure Appl. Math. 2018, 49, 265-284. [CrossRef]

10. Smarandache, F. Neutrosophic set-A generalization of the intuitionistic fuzzy set. Int. J. Pure Appl. Math. 2005, 24, 287-297.

11. Peng, X.; Dai, J. A bibliometric analysis of neutrosophic set: two decades review from 1998 to 2017. Artif. Intell. Rev. 2018. [CrossRef]

12. Peng, X.; Liu, C. Algorithms for neutrosophic soft decision making based on EDAS, new similarity measure and level soft set. J. Intell. Fuzzy Syst. 2017, 32, 955-968. [CrossRef]

13. Peng, X.; Dai, J. Approaches to single-valued neutrosophic MADM based on MABAC, TOPSIS and new similarity measure with score function. Neural Comput. Appl. 2018, 29, 939-954. [CrossRef]

14. Abdel-Basset, M.; Chang, V.; Mohamed, M.; Smarandche, F. A refined approach for forecasting based on neutrosophic time series. Symmetry 2019, 11, 457. [CrossRef]

15. Abdel-Basset, M.; Mohamed, M.; Chang, V.; Smarandache, F. IoT and its impact on the electronics market: a powerful decision support system for helping customers in choosing the best product. Symmetry 2019, 11, 611. [CrossRef] 
16. Broumi, S.; Dey, A.; Talea, M.; Bakali, A.; Smarandache, F.; Nagarajan, D.; Lathamaheswari, M.; Kumar, R. Shortest path problem using Bellman algorithm under neutrosophic environment. Complex Intell. Syst. 2019, 5, 409-416. [CrossRef]

17. Broumi, S.; Bakali, A.; Talea, M.; Smarandache, F.; Dey, A.; Son, L.H. Spanning tree problem with neutrosophic edge weights. Procedia Comput. Sci. 2018, 127, 190-199. [CrossRef]

18. Boloş, M.I.; Bradea, I.A.; Delcea, C. Neutrosophic portfolios of financial assets. minimizing the risk of neutrosophic portfolios. Mathematics 2019, 7, 1046. [CrossRef]

19. Zhang, X.; Bo, C.; Smarandache, F.; Dai, J. New inclusion relation of neutrosophic sets with applications and related lattice structure. Int. J. Mach. Learn. Cybern. 2018, 9, 1753-1763. [CrossRef]

20. Zhang, X.; Bo, C.; Smarandache, F.; Park, C. New operations of totally dependent-neutrosophic sets and totally dependent-neutrosophic soft sets. Symmetry 2018, 10, 187. [CrossRef]

21. Zhang, X.; Smarandache, F.; Liang, X. Neutrosophic duplet semi-group and cancellable neutrosophic triplet groups. Symmetry 2017, 9, 275. [CrossRef]

22. Zhang, X.; Wang, X.; Smarandache, F.; Jaiyeola, T.G.; Lian, T. Singular neutrosophic extended triplet groups and generalized groups. Cogn. Syst. Res. 2019, 57, 32-40. [CrossRef]

23. Zhang, X.; Borzooei, R.; Jun, Y. Q-filters of quantum B-algebras and basic implication algebras. Symmetry 2018, 10, 573. [CrossRef]

24. Zhang, X.; Mao, X.; Wu, Y.; Zhai, X. Neutrosophic filters in pseudo-BCI algebras. Int. J. Uncertain. Quan. 2018, 8, 511-526. [CrossRef]

25. Zhang, X.; Wu, X.; Mao, X.; Smarandache, F.; Park, C. On neutrosophic extended triplet groups (loops) and Abel-Grassmann's groupoids (AG-groupoids). J. Intell. Fuzzy Syst. 2019, 37, 5743-5753. [CrossRef]

26. Ma, Y.; Zhang, X.; Yang, X.; Zhou, X. Generalized neutrosophic extended triplet group. Symmetry 2019, 11, 327. [CrossRef]

27. Shah, M.; Ahmad, I.; Ali, A. On quasi-cancellativity of AG-groupoids. Int. J. Contemp. Math. Sci. 2012, 7, 2065-2070.

28. Ahmad, I.; Ahmad, I.; Rashad, M. A study of anti-commutativity in AG-groupoids. J. Math. 2016, 48, $99-109$.

29. Smarandache, F. Neutrosophic Perspectives: Triplets, Duplets, Multisets, Hybrid Operators, Modal Logic, Hedge Algebras and Applications; Pons Publishing House: Brussels, Belgium, 2017.

30. Khan, M.; Anis, S. An analogy of Clifford decomposition theorem for Abel-Grassmann groupoids. Algebra Colloq. 2014, 21, 347-353. [CrossRef]

31. Wu, X.; Zhang, X. The decomposition theorems of AG-neutrosophic extended triplet loops and strong AG-(1, 1)-loops. Mathematics 2019, 7, 268. [CrossRef] 